\title{
Narrativas civilizatorias de la enseñanza de las matemáticas: lo que se mantiene, irrumpe y se transforma y el sujeto
}

\author{
Civilizational narratives of the teaching of mathematics: what remains, bursts and \\ transforms and the subject
}

\section{Narrativas civilizacionais do ensino de matemática: o que resta, explode e transforma e o assunto}

Néstor Fernando Guerrero Recalde ${ }^{1}$

Recibido: junio de 2018

Aceptado: diciembre de 2018

Para citar este artículo: Guerrero-Recalde, N. F (2019). Narrativas civilizatorias de la enseñanza de las matemáticas: lo que se mantiene, irrumpe y se transforma y el sujeto. Revista Científica, 34(1), 81-100. Doi: https://doi. org/10.14483/23448350.13484

\section{Resumen}

En el presente artículo se muestran los resultados del proyecto de investigación doctoral "Narrativas civilizatorias de la enseñanza de las matemáticas en Colombia". El estudio tuvo como propósito comprender, a partir de narrativas de profesores, las razones que han situado a la enseñanza de las matemáticas como un saber para el progreso en Colombia. Para el análisis de las narrativas de los profesores se empleó la metodología de investigación narrativa propuesta por Quintero (2017). Su carácter hermenéutico-interpretativo reconoce el carácter contextual, inductivo y semántico de la narrativa histórica. Los resultados de esta investigación contribuyen a la reflexión sobre cómo el proceso civilizatorio de la enseñanza de la matemáticas occidentales convirtió a los saberes matemáticos en saberes hegemónicos, subordinando al sujeto a los modelos económicos neoliberales. Frente a esta postura se levanta como alternativa la humanización del conocimiento hacia el cuidado del Otro y de lo otro, en el reconocimiento del pluralismo y de la diversidad de culturas. En las conclusiones del estudio se señala que: en primera instancia, con la enseñanza de las matemáticas se pone en evidencia que la formación que se ha privilegiado en los futuros licenciados, desde la década de 1970 sigue siendo la de las matemáticas modernas. En segundo lugar, que en resistencia al movimiento de las matemáticas modernas irrumpen la perspectiva sociopolítica de la educación matemática. Finalmente, se concluye que a nivel de la formación inicial y continua de los profesores se están dando transformaciones sobre su práctica profesional.

Palabras clave: narrativa, civilización, procesos sociales, sujeto, enseñanza, matemáticas, progreso. 


\section{Abstract}

In the present article the results of the doctoral research project "Civilizing narratives of the teaching of mathematics in Colombia" are shown. The purpose of the study was to understand, from teacher narratives, the reasons that have led to the teaching of mathematics as a knowledge for progress in Colombia. For the analysis of the teachers' narratives, the narrative research methodology proposed by Quintero (2017) was used. Its interpretative-hermeneutic character recognizes the contextual, inductive and semantic nature of the historical narrative. The results of this investigation contribute to the reflection of how the civilizatory process of the teaching of Western mathematics, converted mathematical knowledge into hegemonic knowledge, subordinating the subject to neoliberal economic models. Faced with this position, the humanization of knowledge towards the care of the Other and of the other is raised as an alternative, in the recognition of pluralism and the diversity of cultures. In the conclusions of the study it is indicated that: in first instance, that with the education of the mathematics, it becomes evident that the formation that has been privileged in the future graduates, from the decade of the 70s is still the one of the modern mathematics, secondly, that in resistance to the movement of modern mathematics the sociopolitical perspective of mathematics education breaks out. Finally, it is concluded that at the level of the initial and continuous training of teachers there are transformations on the professional practice of teachers.

Keywords: narrative, civilization, social processes, subject, teaching, mathematics, progress.

\section{Resumo}

No presente artigo são apresentados os resultados do projeto de pesquisa de doutorado "Civilizing narratives of the teaching of mathematics in Colombia". O objetivo do estudo foi compreender, a partir das narrativas dos professores, as razões que levaram ao ensino da matemática como um conhecimento para o progresso na Colômbia. Para a análise das narrativas dos professores, utilizou-se a metodologia de pesquisa narrativa proposta por Quintero (2017). Seu caráter hermenêutico-interpretativo reconhece a natureza contextual, indutiva e semântica da narrativa histórica. Os resultados desta investigação contribuem para a reflexão de como o processo civilizatório do ensino da matemática ocidental converteu o conhecimento matemático em conhecimento hegemônico, subordinando o sujeito a modelos econômicos neoliberais. Diante dessa posição, surge a humanização do conhecimento para o cuidado do Outro e do outro como alternativa, no reconhecimento do pluralismo e da diversidade de culturas. Nas conclusões do estudo indica-se que: em primeira instância, que com a educação da matemática, fica evidente que a formação que tem sido privilegiada nos futuros graduados, a partir da década dos anos 70 é ainda a da matemática moderna em segundo lugar, que, em resistência ao movimento da matemática moderna, a perspectiva sociopolítica da educação matemática se manifesta. Por fim, conclui-se que, no nível da formação inicial e continuada dos professores, há transformações na prática profissional dos professores.

Palavras-chaves: narrativa, civilização, processos sociais, disciplina, ensino, matemática, progresso

\section{Introducción}

La presente investigación tuvo como propósito comprender, a partir de narrativas de profesores, las razones que han Ilevado a situar a la enseñanza de las matemáticas como un saber para el progreso en Colombia. Para dar alcance a este propósito, se establecieron dos objetivos: uno relacionado con la comprensión de los saberes matemáticos que se mantienen, irrumpen y se transforman en procesos de enseñanza; y el otro orientado a describir, en procesos de larga duración, el tipo de sujeto que se busca formar como respuesta a la idea de progreso establecida en la sociedad ${ }^{2}$.

Para analizar la relación entre los cambios sociales y de la personalidad de los individuos, se tomaron en cuenta los planteamientos del sociólogo Norbert Elias, para quien los cambios

\footnotetext{
- Para una mejor descripción de lo que significa el proceso de larga duración en la presente investigación, véase al artículo de Guerrero (2016)
} 
y transformaciones de los sujetos se pueden explicar como un equilibrio entre las restricciones exteriores provenientes de la sociedad y las autorrestricciones del individuo para el control de las emociones en una dirección determinada. Desde esta perspectiva, se puede comprender en un grupo humano o entramado social lo que se mantiene, irrumpe y transforma en relación con procesos sociales $^{3}$. Seguidamente, es posible describir las interdependencias humanas que dan emergencia a un tipo de individuo, en un espacio-tiempo determinado, en procesos sociales de larga duración.

Para describir los procesos de cambio social, se recurrió a la comprensión de los acontecimientos históricos al ubicarlos en especialidades y temporalidades determinadas. Dichos acontecimientos son narrados, en este caso, por los profesores y estudiantes y se refieren a sus experiencias escolares, académicas y pedagógicas en torno a la enseñanza de las matemáticas.

Siguiendo a White (2003), para conseguir estos propósitos, es importante la narrativización, en la medida en que da cuenta de los acontecimientos presentes en un relato. Toda narrativa contiene unos personajes, una historia contada, unos argumentos, una secuencia de acciones e intriga.

Para el análisis de las narrativas de los profesores, se empleó la metodología de investigación narrativa propuesta en el capítulo "Triple mimesis" de Quintero (2017). Su carácter hermenéutico-interpretativo reconoce el carácter contextual, inductivo y semántico de la narrativa histórica. En palabras de Ricoeur (2006), una narrativa se estructura a partir de los procesos de prefiguración, configuración y refiguración de la trama. Desde las voces de los sujetos inmersos en una narrativa histórica, esta intención interpretativa permite

\footnotetext{
Los procesos de cambio social se pueden describir a partir del concepto de cambio direccional significativo de Mennell (2001), siguiendo a Elias (2012), como se afirma a continuación: “Los procesos civilizadores surgen (como procesos ciegos y no planeados) de las luchas de la gente para resolver los problemas que se les plantean en sus vidas mediante presiones desestabilizadoras por ejemplo, la amenaza de violencia e inseguridad" (Mennell, 2001, p. 32).
}

la configuración de tramas desde la experiencia vivida y las concepciones de mundo de vida compartidas. Estas tramas narrativas son reconfiguradas por el lector, lo que aporta a la comprensión de los hechos narrados.

La metodología partió del registro de codificación, para identificar posteriormente en la trama narrativa tres niveles. El primero fue de orden textual y, en su naturaleza descriptiva, buscó dar cuenta de lo que se dice con el lenguaje en aspectos referenciales, tales como hechos, temporalidades y espacialidades. El segundo nivel fue contextual y su centro estuvo en la fuerza narrativa otorgada por el sujeto de la enunciación a sus acciones y atributos relacionados con sus juicios (morales, políticos y retrospectivos), imputaciones, responsabilidades y potencialidades. Esta fuerza narrativa se entiende, según Quintero (2017), como

[...] el uso comunicativo y/o expresivo, a partir del cual hacemos y decimos cosas, lo que implica una correspondencia entre lenguaje y mundo. Fuerzas narrativas que nos indican que en las redes de interlocución, el narrador, si bien tiene un acceso privilegiado, éstas sólo adquieren significado y sentido cuando hacen parte de vínculos comunitarios. (Quintero, 2017, p. 32)

Finalmente, como tercer nivel encontramos el metatexto, que exigió de la polifonía discursiva, la cual dio lugar a la reconfiguración de los niveles textual y contextual.

En el proceso de la recolección de la información se aplicó la entrevista narrativa a cuatro participantes: tres profesores y un estudiante para profesor. Su selección obedeció al criterio del periodo en el que estudiaron, así: dos profesores con experiencia docente en la década de 1980, una profesora sin experiencia docente en esa década y un estudiante para profesor con experiencia docente después de la década de 1990. La sucesión de generaciones se estudia en procesos sociales de larga duración; por ello, la necesidad de enmarcar los acontecimientos en los periodos anteriores. 
A continuación, se expondrán las conclusiones del estudio desde cada sujeto de enunciación, a partir de las narraciones de los profesores Darío $(\mathrm{P}$, $M, 1)$, Marcelo ( $P, M, 2)$, Andrea ( $P, F, 3)$ y Juan (EP, $M, 4)^{4}$. Es preciso señalar que los subtítulos de las conclusiones guardan relación con los objetivos de la investigación.

\section{El sujeto de las matemáticas modernas: formación matemática que se mantiene en procesos de larga duración}

En relación con el sujeto que se pretende formar, cabe resaltar dos aspectos: en primer lugar, la consideración de la idea de figuración - composición, en palabras de Elias - para describir e interpretar los tipos de saberes matemáticos que se mantienen en el tiempo por muchas generaciones — como proceso social de larga duración-y, en segundo lugar, la necesidad de instituciones sociales para el control social.

En este estudio, siguiendo a Elias (2012), entendemos por figuración los entramados sociales que son resultado de composiciones entre individuos que actúan en mutua interdependencia y reciprocidad. Estas figuraciones se constituyen como consecuencia de las presiones sociales provenientes de las instituciones, tales como la escuela que, a partir de los hábitos, costumbres y creencias, modelan el aparato psíquico, estructuran las emociones y permiten el control de la conducta del individuo.

Uno de los principales hallazgos de esta investigación es que los saberes escolares que se mantienen en la enseñanza de las matemáticas es el de las matemáticas modernas. Como manifiesta el profesor Darío:

[hay] avances hacia otras posturas en la formación de profesores, [...] pero creo que se siguen formando en la matemática formal. (P, M, 1, 5-6)

- Se han cambiado los nombres de los profesores participantes y del estudiante para profesor para preservar su identidad en la investigación.
Por su parte, el profesor Marcelo manifiesta que, desde la década de 1970, en los programas de formación de profesores se enseñaban matemáticas modernas por su novedad, actualidad mundial y por su pertinencia en el avance de la ciencia $(P, M, 2)$.

El movimiento de las matemáticas modernas permitió que la sociedad estableciera órdenes sociales y políticos para promover la adaptabilidad de los sujetos. Como señala el profesor Darío:

Uno encuentra que hay análisis que hablan [de] que la escuela es un espacio básico para acostumbrar al sujeto, para luego encajar en el sistema económico productivo, Ilámese fábrica, llámese empresa, Ilámese actitudes de obediencia; para seguir normas $y$ reglas. $(P, M, 1,59-61)$

Es decir, desde la escuela, a través de las matemáticas modernas, se instauró un orden social en el que se adaptaba, pero también se nutría la idea de formar un sujeto para el capitalismo.

El sujeto que se quiere formar con las matemáticas modernas orienta sus comportamientos y conocimientos hacia la profesionalización propia de ciencias aplicadas como la ingeniería, la economía o las finanzas. Recordemos que estas áreas se consideran los pilares del progreso en nuestro país y que se han formado bajo el supuesto de que aportan en el avance de la ciencia. Para ello, se toman como ejemplo otros países en vía de desarrollo como Chile y Brasil. Esta enseñanza se fundamenta en la idea de que el proceso civilizatorio conduce al progreso social y, con ello, a la aspiración de un hombre ilustrado, culto y perfecto que es artífice de los procesos de industrialización y revolución tecnológica.

Las matemáticas modernas no se han dejado de enseñar. Lo expuesto coincide con Hernández (2000), cuando señala cómo con las matemáticas modernas, en los países desarrollados, se impulsa la idea de que solo el manejo de la técnica permitirá el progreso de la ciencia frente a las sociedades con menor desarrollo científico: "El miedo a 
quedarse atrás a causa de una educación científica y técnica inferior cundió y tal vez contribuyó a facilitar lo que de otro modo hubiera sido más arduo o imposible. Algunos matemáticos importantes intervinieron en los cambios" (Hernández, 2000, pp. 299-300). Esta idea de progreso científico con las matemáticas modernas se relaciona con lo expuesto por Elias sobre evolución social por "aquello que cada sociedad expresa en su peculiaridad y de lo que se siente orgullosa representado en el grado alcanzado por su técnica, sus modales, el desarrollo de sus conocimientos científicos, su concepción del mundo y muchas otras cosas" (2012, p. 57).

Una manifestación de esta evolución la encontramos en las narrativas de los profesores cuando señalan que la formación que se privilegiaba en los futuros licenciados era la de las matemáticas modernas (formalistas), una estrategia fomentada en todos los currículos del mundo y en Colombia con el propósito de poner a los países a la vanguardia de la revolución tecnológica. Estas matemáticas se fundaban sobre la lógica formal, los modos de razonamiento deductivo y los métodos de demostración matemática. A partir de esta formación, el estudiante se aproximaba a la idea de una realidad dada, de verdades que son consistentes en sí mismas, del rigor y del poder de la axiomatización que tienen las estructuras matemáticas. Señala en su narrativa el profesor Marcelo, uno de los participantes del estudio, que se trataba de unas matemáticas en las que el individuo no cabía, como tampoco el mundo concreto ( $\mathrm{P}, \mathrm{M}, 2)$.

En consonancia con este hallazgo, en la época en que se impuso este movimiento, según Hernández (2000), se hablaba de una enseñanza orientada hacia la abstracción pues representaba progreso de la ciencia matemática. Como precisa Sánchez (2012), las viejas matemáticas, las del siglo XIX, habían representado para nuestro país un atraso en la formación de los matemáticos profesionales y los profesores de secundaria. De este modo, los esfuerzos del país se orientaron a impulsar reformas que se pusieran a tono con los avances en países más desarrollados y adoptaran tendencias para modernizar las ciencias, como sucedió en la educación colombiana.

No obstante, las resistencias de los profesores frente a este enfoque llevaron a comprender que las matemáticas modernas estaban alejadas de la realidad social y que además constituían un modo de control desde la técnica, pues los instrumentos que acompañaban su uso se vinculaban con la idea del optimismo tecnológico. Las matemáticas modernas, señala el profesor Darío, son usadas:

"como instrumento tecnológico para acceder a unos conocimientos básicos para que produzca para el modelo económico y también la institución escuela, clase de matemáticas, como mecanismo para formar sujetos para ese modelo" (P, M, 1, 62-64).

En otras palabras, se trataba de unas matemáticas que encajaran en el sistema productivo y se orientaran a la generación de bienes y la obtención de bienestar, entendido este como consumo, tener capital, comprar y vender servicios.

Además, el profesor Marcelo es enfático al afirmar que este tipo de formación matemática pudo ser pertinente para el matemático puro o para el ingeniero, pero no para el profesor. Esta idea se vincula con lo expuesto por Goñi (2010) cuando señala lo desacertado de esta postura en la enseñanza de las matemáticas: "[...] querer pensar que todos los ciudadanos hagan matemáticas como los matemáticos profesionales es un desatino, una exageración y que los estudiantes lo hagan con la lógica formal fue para la época una idea traída de los cabellos" (p. 20). Se puede decir que, con los saberes de la matemática moderna, no es posible desarrollar el pensamiento del niño o el joven al nivel de un matemático.

Como afirma Rojas (2001): "El deseo civilizador significaba acceder a una civilización ordenada como la europea, y corrió paralelo con la lucha por la acumulación de capital". Bajo este precepto, los niños y jóvenes deben formar su espíritu científico para contribuir con el desarrollo 
tecnológico y científico del país. Es decir, se difunde la idea de que estas matemáticas contribuyen al desarrollo intelectual: el que sabe matemáticas es inteligente, capaz, talentoso, creativo, etc.; el dominio de la técnica por los matemáticos o los profesionales con carreras afines los vuelve aptos para participar del progreso social, económico y científico en las sociedades.

Para los profesores que hicieron parte de la presente investigación estas ideas civilizatorias y del progreso generaban expectativas sociales en la gente común y entre los profesionales de otros campos. Expectativas que también tenían quienes decidían estudiar para ser profesores. Cuando los estudiantes están en proceso de formación para ser profesores, sus expectativas recaen, por ejemplo, en ver más contenidos y menos conceptualización. El estudiante Juan manifiesta que esperaba más clases para aprender técnicas de cálculo y creía que su formación matemática era suficiente para enseñar. También señala Andrea que, entre quienes se forman como futuros licenciados, se piensa que saber matemáticas les va a dar un mayor prestigio, porque consideran que van a ser matemáticos y no profesores $(\mathrm{P}, \mathrm{F}, 3)$.

Entre las conclusiones del trabajo de Gómez (2010), que retomamos en el caso de las matemáticas modernas como hechos ilustrativos de la problemática anterior, encontramos que: a) los inconvenientes de la enseñanza de la matemática moderna en el bachillerato fueron causados por la falta de preparación de los profesores; b) existió una dificultad en los estudiantes al asimilar esta nueva forma de presentar la matemática; c) un currículo de matemática en primaria no presentó avances significativos en el aprendizaje de esta matemática (p. 173). En consecuencia, podemos decir que estos hallazgos coinciden con lo que señalan los profesores, pues los estudiantes no tenían las herramientas cognitivas para abordar estos contenidos y se produjo una ruptura entre los modos de enseñanza fundamentados en la repetición y los que se exigen en la lógica formal. Tal como plantea el profesor Darío:
"En el colegio todo era completamente mecánico; para luego tratar de encontrar el fundamento, la lógica que subyace, ya no en la ejecución del algoritmo sino en el trasfondo matemático desde la teoría de conjuntos y sus métodos lógicos". (P, M, 1, 20-21)

Con la implementación de las matemáticas formalistas, como las matemáticas modernas, en nuestro país es posible ver su incidencia en la formación en el largo plazo. Esto se puede apreciar tanto en lo que se juzga como formación básica de un estudiante de primaria y secundaria como en lo que se supone que debe saber un profesor para enseñar. Tal como manifiesta la historiadora de las matemáticas Sánchez (2012), la tradición del enfoque de las matemáticas modernas en Colombia se ha mantenido desde mediados de la década de 1960, primero con la formación en las normales superiores y después con su introducción en la educación primaria y secundaria, a través de la Universidad Pedagógica Nacional en Bogotá y la Universidad Pedagógica y Tecnológica en Tunja, donde se formaba a los licenciados (p. 134).

La explicación de Elias (2012) a esta problemática educativa es que, a medida que se incrementan las presiones sociales provenientes de los Estados, grupos e instituciones sociales, como universidades, colegios e incluso la familia, en los que recae la responsabilidad de civilizar o educar por medio de la técnica el comportamiento, las emociones y la personalidad, se intensifican en el individuo las autorrestricciones y se consigue la adaptabilidad social. En su narrativa, Darío relata que se solía aprender matemáticas (fundamentos de matemáticas) con profesores autoritarios y crueles, quienes, en su caso, le generaron frustraciones que pudo superar "con mañita", con la ayuda de sus compañeros y a través de tutorías $(P, M, 1)$. De esta manera, el modo en que el profesor enseña estas matemáticas se considera clave para generar habitus y creencias en el estudiante. Retomando a Elias (2009), el aprendizaje debería corresponder con aquel individuo capaz de entender con el uso de la razón, el lenguaje y la acción. 
Para los profesores participantes de esta investigación, las matemáticas modernas no solo hicieron explícita la intención de civilizar para cambiar las estructuras cognitivas de los aprendices, sino también se consideraban la solución a los problemas de pensar el desarrollo en las sociedades capitalistas para dividir a las sociedades entre pobres y ricas. A partir de Elías (2008) se puede sostener que, a grandes rasgos, se trata de reducir la pobreza relativa de esas sociedades (p. 173).

Podemos afirmar que, en sociedades con menor grado de evolución social, con menos progreso en la ciencia se da una estratificación y distribución de los saberes entre clases sociales, entre los que van a gobernar y los gobernados. Entre unas matemáticas para los obreros, campesinos, asalariados, negros, etc. y otra para las élites con mayor grado de sofisticación, para la dirección y administración de los flujos de capital.

Para facilitar el tránsito al capitalismo a través de la educación obligatoria, tal como señalan los profesores en sus narrativas, la reforma de las matemáticas modernas superó la vieja usanza. Las viejas prácticas estaban orientadas hacia el enciclopedismo académico y, por tanto, carecían de los fundamentos cognitivos respecto al sujeto que aprende, que es el caso tanto de los que van a ejercer la profesión 'ser profesor' de matemáticas, como de los mismos formadores y como del aprendizaje de los niños y jóvenes estudiantes. En palabras del profesor Marcelo, eso sucedió con los profesores que se formaron en las facultades de ingeniería o en las facultades de matemáticas y fueron los primeros profesores en los programas de formación de licenciados $(P, M, 2)$.

Se pensaba que la enseñanza de las matemáticas modernas facilitaría durante el proceso instructivo aprender a razonar, inferir y trabajar como el matemático profesional, con el pensamiento abstracto-reflexivo, a partir del rigor lógico y la demostración. Pero como se puede entrever con las palabras de Andrea en su narrativa, la utilidad de las matemáticas formalistas modernas en la formación del sujeto (esas que "están allá") se reduce a los asuntos de lo escolar, a pasar una asignatura $(\mathrm{P}, \mathrm{F}, 3)$. Por ello, teniendo en cuenta lo dicho por Gómez (2000), el énfasis en el aprendizaje sigue centrándose en la formación tradicional, en la mecanización y memorización de los contenidos curriculares.

La sociedad colombiana, entonces, propuso la reforma de las matemáticas modernas que, como movimiento educativo, tenía entre sus pretensiones formar al individuo para el trabajo práctico, para el dominio de habilidades tecnocientíficas, para contribuir con el progreso social y de la ciencia. Elias (2012) ilustra esta formación como necesaria para el proceso de industrialización, para la emergencia de entramados profesionales con vínculos sociales y científicos, de versatilidad y especialización en el manejo de la técnica.

De esta manera, durante la formación del sujeto, no se producen rupturas ni discontinuidades. Por ejemplo, Andrea narra que, después de haber pasado por muchas experiencias educativas, ella se dio cuenta de que las experiencias con las matemáticas en la universidad son las mismas que las del colegio, en cuanto a cómo se aprenden y se enseñan $(\mathrm{P}, \mathrm{F}, 3)$. ¿Existe una continuidad entre cómo se comprende y se otorga significado sobre qué tipo de matemáticas? ¿Para qué tipo de sujeto? ¿Para qué sociedad? Se trata de las matemáticas para el sujeto de la modernidad. Por lo tanto, se sigue con la enseñanza de las matemáticas modernas; solamente hay algunos esbozos de cambio.

De acuerdo con Arboleda (2015), al haber nacido la profesión de 'ser profesor' junto a la de matemático e ingeniero, desde el siglo XIX en Colombia, y con el desarrollo de la matemática moderna en todas las universidades del país, se consideró el saber matemáticas como saber totalizador de la experiencia docente. Esta postura se vincula con lo que manifiesta el profesor Marcelo en su narrativa al afirmar que no solo se crearon falsas expectativas en la sociedad sobre el rol del profesor, sino que se le ha entregado a los programas de formación la tarea de resolver el problema de garantizar que se produzcan las conexiones 
entre los saberes didácticos y los saberes de referencia disciplinar de las matemáticas, tanto en lo que concierne a la práctica docente como también a la práctica matemática $(\mathrm{P}, \mathrm{M}, 2)$. En este sentido, los profesores seguiremos creyendo que buena parte de los problemas de la didáctica radica en verla exclusivamente como un conjunto de preceptos para actuar en el aula y no como una posibilidad de transformación de las subjetividades desde los mismos estudiantes, quienes son a la vez aprendices, prácticos y ciudadanos.

Uno de los hallazgos de la presente investigación respecto al proceso civilizatorio (formación del sujeto en estructuras sociales) fue precisamente considerar que, con la reforma de las matemáticas modernas, se hacen explícitos procesos sociales de larga duración y cómo influyeron en mantener con la enseñanza de las matemáticas un tipo de sujeto a formar. Este es el movimiento educativo más importante que ha permeado todos los niveles de la educación - desde la primaria y la secundaria hasta la universidad-.

En las voces de los profesores y de los estudiantes para profesor, dichas manifestaciones se presentan en los siguientes rasgos:

a. Las bases matemáticas de los estudiantes los faculta para pensar con la técnica (EP, M, 4).

b. Se sigue formando a los futuros licenciados en las matemáticas modernas y se agrega lo didáctico y pedagógico. Una formación clásica $(\mathrm{P}, \mathrm{F}, 3)$.

C. Las matemáticas siguen discriminando entre el que sabe y el que no sabe. Las matemáticas dan poder (EP, M, 4).

d. La cuestión de las matemáticas pasa por ser una asignatura para aprobar, lejos de la vida de los estudiantes y que está alejada de la realidad ( $\mathrm{P}, \mathrm{F}, 3)$.

e. Las matemáticas modernas son descontextualizadas de su medio, de su entorno inmediato, de la vida cotidiana ( $\mathrm{P}, \mathrm{M}, 2)$.

f. No se puede enseñar matemáticas sin matemáticas formales (P, F, 3). g. Las matemáticas modernas eran el saber de referencia del saber del profesor y del matemático puro (P, M, 2).

h. En la escolaridad normal no se encuentran aplicaciones de esas matemáticas a la vida cotidiana $(\mathrm{P}, \mathrm{F}, 3)$.

i. Matemáticas que servían para asuntos de lo escolar (P, F, 3).

j. La enseñanza en los primeros semestres era netamente disciplinar, eran matemáticas formales (P, F, 3).

k. La formación sigue siendo en las matemáticas formalistas ( $P, M, 1)$.

I. Se sigue pensando en el desarrollo del pensamiento matemático por el pensamiento $(\mathrm{P}, \mathrm{M}, 1)$.

$m$. Los profesores que enseñaban bajo este enfoque no tenían la formación para hacerlo $(\mathrm{P}, \mathrm{M}, 2)$.

Estos rasgos nos dejan ver que estas matemáticas, en el largo plazo, durante varias generaciones en el periodo estudiado, no solo produjeron resistencias frente a su enseñanza por parte de profesores, investigadores, estudiantes de licenciaturas, padres, niños y jóvenes, sino que además demandaron dinámicas educativas de apropiación de estos saberes, que implicaron una fuerte escisión entre quienes consideraban que era suficiente para enseñar y los que creían que sin una conexión con los saberes pedagógicos y didácticos esto no era posible. Como señala el profesor Darío, se trata de unas matemáticas que estructuran el pensamiento lógico racional en los jóvenes, pero que no se acomodan a los conocimientos y formas de razonar de los estudiantes (P, M, 1).

Como nos recuerda García (1996), estas 'nuevas matemáticas' "[...] confundieron y produjeron rechazo en estudiantes y padres de familia quienes se quejaron por no poder ayudar a sus hijos en las tareas, y la escasa preparación de los profesores pues los cursos de capacitación ofrecidos fueron insuficientes" (p. 197). Esto marca una distinción entre lo que debe enseñarse en la educación obligatoria y la universidad, entre lo que debe saber un profesor para enseñar y lo que es el 
currículo obligatorio enfocado hacia estos saberes matemáticos.

\section{Matemáticas para la resistencia y para la transformación de la sociedad}

En atención al objetivo de comprender los saberes matemáticos que se mantienen, irrumpen y se transforman en los procesos de enseñanza de las matemáticas, a continuación, se presenta el desarrollo de la conclusión sobre los hechos que allí irrumpen.

En la enseñanza de la matemática en Colombia las reformas y tendencias pedagógicas que se implementaron para cambiar las viejas prácticas hegemónicas establecidas por la enseñanza de las matemáticas modernas fueron infructuosas, pues no se sostuvieron en el tiempo. Por ello, no se puede juzgar como un proceso de larga duración, pero se puede considerar entonces que han irrumpido sin producir los cambios educativos anhelados.

Ante lo hegemónico surgen nuevas resistencias. Estas dan lugar a otros modos de pensar y proponer la enseñanza de las matemáticas. Como señalan los profesores en sus narrativas, de estas formas de resistencia social han emergido dos posturas que, si bien tuvieron eco entre los investigadores y formadores, se han desarrollado escasamente para la transformación de las prácticas docentes de los profesores.

Por una parte, se encuentra la orientación hacia posturas críticas en educación matemática, sustentadas desde visiones sociales y políticas, como en el caso de Valero y Skovsmose, y ampliamente difundidas en América Latina. Para estos autores, la construcción de los saberes matemáticos pasa por la revisión de la pregunta para qué se enseña determinado concepto y cuáles son sus implicaciones en el ejercicio de una ciudadanía activa en una sociedad democrática. Esta se puede considerar la primera irrupción en nuestro contexto colombiano.

Otro hallazgo de la investigación, que se hace explícito en las narrativas de los profesores, es que, como resistencia a las matemáticas modernas, se dio un giro decolonialista y contrahegemónico, desde el cual se reconoció que en las culturas se producen saberes matemáticos distintos a los de Occidente. Esto corresponde con lo que en educación matemática se conoce como el enfoque de las etnomatemáticas, cuyo exponente principal es el investigador D'Ambrosio. Esta postura en nuestro país constituye la segunda irrupción. En lo que sigue, se presentan las conclusiones que emergen con cada una de estas irrupciones, a través de lo que develan los docentes en sus narrativas.

\section{Matemáticas y ciudadanía: demandas y exigen- cias en la formación del sujeto ético y político}

Para los profesores y el estudiante de licenciatura en formación que hicieron parte de la presente investigación, la enseñanza de las matemáticas debe estar relacionada con la formación de un sujeto crítico. Para el profesor Marcelo, es la manera como se asume una posición política frente al mundo contemporáneo con las matemáticas para enfrentar la realidad social, para participar en la construcción de una sociedad democrática $(\mathrm{P}, \mathrm{M}, 2)$. Esta propuesta se relaciona con la postura de Skovsmose y Valero (2012) cuando señalan que

[...] la enseñanza de las matemáticas debería ayudar a que los estudiantes experimenten y reconozcan el papel de las matemáticas en la sociedad y en la cultura. El objetivo de esta postura es que los estudiantes sean capaces de tomar responsabilidades y de participar en una comunidad democrática, deberían poder comprender las maneras en que las matemáticas se usan. (Undervisningsministeriet, 1995, p. 9, citado en Skovsmose y Valero, 2012, p. 2)

Los estudiantes deberían comprender que las matemáticas no son ideológicamente neutras y libres de valores, tal como se instauró con la modernidad. Se puede concluir desde lo dicho por los profesores en sus narrativas que el sujeto puede 
entonces asumir posturas críticas sobre una matemática que genera inequidad, injusticia y desigualdad. Esto implica la asunción de valores para la vida democrática. Como señalan Skovsmose y Valero (2012), al referirse al papel de la enseñanza de las matemáticas para empoderar a los ciudadanos en el vivir democrático: "Si los niños reciben una buena enseñanza matemática, esto les enseñará mucho de la libertad, las habilidades y, desde luego, las disciplinas de expresar, disentir y tolerar que necesita la democracia para tener éxito" (Hannaford, 1998, p. 186, citado en Skovsmose y Valero, 2012, p. 4).

Los profesores reconocen que aunque hay avances en la investigación en educación matemática, en asuntos relacionados con matemáticas y sociedad, se requiere un salto cualitativo hacia perspectivas más contrahegemónicas. Esto con el fin de que no se prescriba una didáctica centrada en el ideal de una formación del sujeto independiente de los contextos sociales y políticos, ajena a nuestra realidad social y cultural colombiana y latinoamericana.

Esta conclusión se puede enmarcar en lo que Skovsmose y Valero (2012) denominan investigación en educación matemática internalista. Según estos autores, esta se sustenta por "[...] el desarrollo de programas de investigación que salvaguardan sus preguntas de investigación de la 'contaminación' de la sociedad y de la política" (p. 6). Tal es el caso de enfoques como la didáctica fundamental francesa, que tanto auge ha tenido en nuestro país pero que, aunque refiere el sistema didáctico y la ecología del aula, no toma en cuenta la realidad de las aulas colombianas.

Por otra parte, en las narrativas de los profesores se considera que las perspectivas sociales en educación matemática no han permeado los currículos en los distintos niveles de la formación del sujeto $(P, M, 1)$, especialmente en lo que tiene que ver con los futuros licenciados, ya que se mantiene la formación como antaño. Los docentes sienten que el esfuerzo de pensar por un tipo de sujeto que ejerza su ciudadanía activamente en nuestro país se ve abocado al fracaso, pues es mayor el peso de la tradición sobre las prácticas de aula que naturalizan las matemáticas como instrumento para el progreso. Estos planteamientos reflejan lo que Skovsmose y Valero (2012) señalan en relación con las reformas en la enseñanza de las matemáticas cuando afirman que:

Hemos sido testigos de diferentes intentos de reforma de la enseñanza de las matemáticas, con diferentes objetivos; sin embargo, la justificación básica de la enseñanza y el aprendizaje de las matemáticas —en términos de asociación al desarrollo tecnológico y, por consiguiente, al progreso social y al bienestar- no ha cambiado en esencia en los últimos cincuenta años. (p. 4)

Otro punto que emerge de las narrativas es que durante la escolaridad nos han mostrado una sola matemática, que se nos impone con un currículo único, con una enseñanza que discrimina al que sabe del que no sabe, al ilustrado del iletrado, para instaurar un saber que jerarquiza en las clases sociales, pero que homogeniza.

Entre las premisas que se expresan en las voces de los profesores y representan distintas generaciones sobre la formación del sujeto están las siguientes:

a. Las matemáticas, desde los ámbitos de la ética, la convivencia y la ciudadanía, se deben enseñar para transformar las realidades sociales $(\mathrm{P}$, $M, 1)$.

b. La matemática anticapitalista posibilita la construcción social de mundos posibles ( $P, M, 1)$.

c. Con la matemática pluralista se pueden hacer cambios sociales desde lo que la gente sabe, desde las culturas (P, M, 1).

d. La resistencia social desde las matemáticas; son muchas manos trabajando juntas desde distintas orillas (P, M, 1).

e. Se deben orientar las políticas públicas de formación en una dirección distinta a la dirección de la hegemonía cultural del Estado (P, M, 2). 
f. La reflexión sobre la práctica docente en la formación de los licenciados hace a los profesores críticos (P, F, 3).

g. Los programas de licenciatura tienen el compromiso de impactar la formación matemática de las generaciones futuras $(\mathrm{P}, \mathrm{F}, 3)$.

h. El problema de la enseñanza de las matemáticas está vinculado con el sentido de los saberes, de lo que es posible aprender y enseñar (EP, M, 4).

Se hace explícito en las narrativas de los profesores que, entre las comunidades de educadores matemáticos, en los programas de formación, en los congresos y encuentros de investigadores, cada vez está tomando más fuerza la asunción de socioepistemologías, pues en los procesos de construcción de conocimientos en las aulas se está considerando el hacer matemático como una práctica social y, por tanto, como una acción política. Por lo anterior, es necesario que los futuros profesores asuman una postura política frente a qué matemáticas enseñar, por qué y para qué. Estas demandas coinciden con lo señalado por Vanegas y Giménez (2011), para quienes el desarrollo de una identidad profesional en los futuros profesores depende de que ellos interpreten la actividad matemática como transformadora, inclusiva intercultural y abierta para la formación en la autonomía y creatividad científica (p. 2).

Puede entonces concluirse, como expresa en su narrativa el profesor Marcelo, que la actividad matemática está rodeada de un conjunto de ideales y valores. Una formación humanística del sujeto desde las matemáticas deberá tomar en cuenta que los ideales son distintos para cada cultura para el matemático, campesino, profesor, negro, obrero, ama de casa, etc. - y que deben inscribirse en un sistema de valores ético-políticos para la inclusión, en el cual se reconozca la diversidad y pluralidad ideológica y se respete la soberanía de los pueblos. Así mismo, deberá poner sobre el pedestal la singularidad de la experiencia humana de la formación del sujeto. Estos ideales y valores de la actividad matemática se pueden enmarcar en la propuesta de Vanegas y Giménez (2011) sobre el desarrollo de la competencia ciudadana a través de las matemáticas, cuando señalan que

[...] como docentes promovemos la competencia ciudadana a través de las matemáticas en cuanto fomentamos (docentes y estudiantes) un conjunto de saberes y prácticas matemáticas reflexivas comprometidas, responsables y solidarias, mediante el desarrollo del pensamiento matemático crítico y toma de conciencia del papel ético de hacer matemáticas, con el fin de aprender a reconocer el valor de construir matemáticas para interpretar hechos y cambios sociales, y aprender participar democráticamente en procesos decisorios comunitarios. (p. 2)

Se trata, entonces, que en las propuestas de formación de profesores, durante la enseñanza y el aprendizaje de los más jóvenes (futuros conciudadanos), se adopte una postura que posibilite el desarrollo de prácticas matemáticas personalizadas y contextualizadas desde nuestra realidad social.

Del mismo modo, los profesores en sus narrativas expresan que la utopía del cambio social podría alcanzarse si se democratizan los saberes matemáticos en las aulas y se posibilita tomar una posición política frente al mundo. Esta manera de entender la realidad social con las matemáticas nos lleva a concluir, en palabras de Skovsmose y Valero (2012), que:

[...] llega a ser esencial para el trabajo de investigación y desarrollo ocuparse de cómo la educación matemática puede asegurar la equidad y la justicia. ¿De qué manera puede la educación matemática, como parte de un sistema educativo nacional, reaccionar contra la reproducción de las desigualdades sociales? (p. 21)

Estas nuevas agendas han sido motivo de debate en el pasado reciente, pues la coyuntura de la política educativa actual lo permite. En épocas anteriores, como sostiene el profesor Marcelo, los 
profesores y estudiantes no tenían los saberes pedagógicos ni didácticos que les permitieran vincular a las matemáticas con procesos sociales. Eran "resistencias espontáneas"; no se comprendía la naturaleza social de los conocimientos matemáticos, de lo que se necesita para tomar conciencia política del deber ser en la formación del sujeto.

Entre los profesores entrevistados se comparte la idea de que la enseñanza de las matemáticas no estaba conectada con las transformaciones sociales, por la falta de cohesión entre las comunidades educativas y los profesores. Esta problemática no ha tocado los intereses de los formadores en las universidades, ya que las comunidades autónomas de profesores no se ponen en diálogo con las autoridades nacionales, de cara a constituir una agenda para las nuevas orientaciones de la educación matemática, o porque el nivel de las prácticas no ha sido permeado por los nuevos paradigmas sobre inclusión intercultural en las aulas. Y esto se sigue manteniendo en el presente. Para el profesor Darío, por ejemplo, es necesario en la actualidad cuestionar la afirmación de "formar profesores de matemáticas críticos" (P, M, 1) o, como manifiesta Andrea, se tienen buenas intenciones, "pero que haya una matemática que contribuya al cambio de realidades, no hay" (P, F, 3, 106-107).

\section{Matemáticas e interculturalidad: demandas y exi- gencias para la formación del sujeto en diversos contextos culturales}

Otro hallazgo importante, que está vinculado con la manera como los profesores asumen una postura política con las matemáticas, también está presente en las narrativas cuando se manifiesta la necesidad de promover la formación de profesores de matemáticas para hacer resistencia social a los modelos económicos que han traído exclusión, dominación y subordinación de los saberes en las culturas no occidentalizadas. Para ello, los profesores proponen una visión de las matemáticas que corresponda a dinámicas socioculturales nuestras, a sistemas de prácticas matemáticas propias.
En particular, se refieren al giro que está tomando la investigación didáctica para incluir dentro de sus análisis las prácticas matemáticas de diversos contextos sociales y culturales. Además, como señala el profesor Marcelo, tenemos:

Derecho a pensar en epistemologías propias de nuestro devenir y de la manera en que nos hemos construido acá y que por consiguiente, las subjetividades pueden responder a esas organizaciones de verdades propias de nosotros, sino seguiremos insistiendo en que solo hay una epistemología universal. (P, M, 2, 210-212)

En estos hallazgos se evidencia que entre los enfoques que han favorecido a los profesores para la toma de posición crítica sobre esta problemática educativa, y que ha irrumpido en la enseñanza de las matemáticas, se encuentran las etnomatemáticas. Estas han permitido comprender con el tiempo cómo son los saberes matemáticos de distintas culturas, sus usos, instrumentos, etc., para tender puentes entre los saberes occidentales y las prácticas interculturales. En esta dirección, Skovsmose y Valero (2012) definen las etnomatemáticas como un programa de investigación que se ocupa de la conexión entre las matemáticas como práctica cultural integrada y las matemáticas como un sistema científico institucionalizado (Powell y Frankenstein, 1997, citado en Skovsmose y Valero, 2012, p. 18).

Se encuentra que, para los profesores, las etnomatemáticas buscan reconocer la diversidad de prácticas matemáticas que se pueden dar en nuestro contexto sociocultural y que, por esta razón, se deben incluir en el aula regular; sobre todo la diversidad de representaciones culturales de nuestros propios estudiantes —miembros de sectores como las negritudes, campesinos, obreros, comerciantes, entre otros-. En consecuencia, señala el profesor Marcelo:

Hay que hacer una fractura en el medio, en el patrón epistemológico que nos viene del norte. $(\mathrm{P}, \mathrm{M}$, 2, 199-200) 
Es decir, con lo que usualmente conocemos como las matemáticas escolares, las que se prescriben en el currículo oficial. Una ruptura con saberes matemáticos hegemónicos, como las matemáticas formalistas, que implique acceder a distintas maneras de construir la realidad; para comprender y conocer otras maneras de enseñar matemáticas, más inclusivas, que representan a todas las culturas, junto con la eurocéntrica $(P, M, 2)$.

Podemos enfatizar en esta postura que, de acuerdo con D'Ambrosio (2007) desde la perspectiva de las etnomatemáticas, las matemáticas occidentales son una de tantas matemáticas, no la única, que con el correr del tiempo se institucionalizó y se consideró como un saber hegemónico; que se uniformizó y universalizó como lo verdadero, dado y establecido. Para enfrentar el problema de la colonialidad del saber occidental, propone D'Ambrosio (2007) "[...] hacer de la matemática una disciplina que preserve la diversidad y elimine la desigualdad discriminatoria, importante para una matemática de carácter humanístico" (p. 102). Por diversidad se puede entender en este contexto estar en armonía con el medio ambiente, con los demás, con uno mismo. Estas matemáticas deben fomentar el respeto a las diferencias y privilegiar el reconocimiento del Otro como un igual, para no violar su identidad cultural en el conjunto de sus valores, costumbres, creencias y cosmovisiones.

Los profesores señalan que, en relación con los saberes matemáticos de otras culturas, hasta hace poco se consideraba que los que no se ajustaban al modelo newtoniano del conocimiento eran considerados saberes de influencia perniciosa, primitivistas. Contrario a ello, señalan que se podría:

Pensar qué tipo de lógicas hay allí, en la manera en la que el estudiante está tratando de supuestamente oponerse a ese obstáculo epistemológico, a lo Otro.

(P, M, 2, 206-207)

Al desconocer las llamadas prácticas matemáticas "primitivistas", "ancestrales" o "empíricas" la escuela ha excluido a los miembros de las diversas culturas, del mundo académico escolar, y ha impedido que ellos ejerzan su ciudadanía en igualdad de condiciones que el resto de sus conciudadanos. Como señala D'Ambrosio (2000): “El rechazo y exclusión de las culturas de la periferia, tan común en el proceso de colonización, todavía prevalece en la sociedad moderna. Grandes sectores de la población no tienen acceso a una ciudadanía completa" (p. 440). En otras palabras, permanece la idea de unas matemáticas para la élite y otras para los subordinados (bárbaros, incultos e iletrados).

En conclusión, se trata de formar ciudadanos con competencias matemáticas que los empoderen como sujetos de derechos y que incluyan dentro de sus saberes los conocimientos e instrumentos de su cultura. Como afirma Juan, se pretende que:

Los estudiantes sean capaces de crear sus propios contextos. (EP, M, 4)

Que vean que las matemáticas son una actividad humana corriente, necesaria para modelar los fenómenos que estudian los científicos, los que le enseñan en la escuela, así como también los de su vida cotidiana.

Para el profesor Marcelo, el problema que tienen entre manos el didacta y el pedagogo sobre las matemáticas inclusivas es cómo:

Tratar los saberes matemáticos para que puedan entrar en la dimensión subjetiva de distintas culturas representadas en el aula (P, M, 2, 223-224).

Es imposible prescindir de la matemática occidental, pues nuestra cultura está sostenida sobre estos saberes. No obstante, se requiere ampliar la visión hacia los saberes ancestrales, empíricos, de grupos marginales como campesinos, obreros, de oficios prácticos, entre otros.

Este tratamiento debe asumirse desde una postura ética durante la formación del sujeto para lograr que con los saberes matemáticos occidentales 
no se produzca la exclusión. Esto se vincula con lo que D'Ambrosio (2007) sostiene respecto a que "[...] el alumno pierde sus raíces culturales, parte de su identidad, que durante el proceso se ven eliminadas" (p. 99). Dicha posición coincide con la narrativa del profesor Marcelo, quien señala que no es posible interpretar o leer estas prácticas matemáticas de otras realidades desde nuestras matemáticas escolares. Tenemos el imperativo ético de aprender de estas prácticas; debemos saber intervenirlas, reconocer lo propio de cada situación matemática, cómo se usan y para qué.

Esta manera de comprender las matemáticas en las culturas, como lo propone D'Ambrosio (2000), trae consigo la exigencia de hacernos responsables de construir los requerimientos para sentar las bases de una nueva civilización que incluya entre sus saberes las técnicas, lenguajes, simbologías de todas las culturas, así como las costumbres, hábitos y creencias para la constitución de nuevas subjetividades. Con esta visión etnomatemática se conseguirá

[...] rechazar la falta de equidad, la arrogancia y el fanatismo que detentan las matemáticas occidentales al considerarse saber universal a todas las culturas. Solo se puede lograr esta nueva civilización a través de la redención de las culturas que han sido subordinadas por mucho tiempo, dando prioridad al fortalecimiento de los sectores de sociedades excluidos [como los bárbaros, incultos e iletrados]. (p. 439)

Esta redención es resultado de las resistencias sociales a los saberes que se imponen, que buscan emancipar al sujeto, para la construcción de una matemática para todos.

Entre los profesores hay acuerdo en que estos análisis no han pasado por las aulas de los profesores formadores para concientizar a los futuros licenciados sobre la necesidad de conocer esas otras realidades en sus contextos sociales y se comprometan con agendas políticas para la inclusión social. Como manifiesta en su narrativa el profesor Marcelo:
Los educadores matemáticos están abriéndose a estas reflexiones, por lo menos; es una reflexión académica y no práctica como debería ser, que de la preocupación se pase a la toma y creación de instrumentos y dispositivos para convertir esa reflexión en acciones concretas. (P, M, 2, 233-235)

Marcelo concluye que en la formación inicial de los profesores, durante sus prácticas de aula, se deben implementar aprendizajes a partir de prácticas matemáticas inclusivas que emerjan del tratamiento y conocimiento de las matemáticas en contextos socioculturales propios.

Las afirmaciones del profesor Marcelo están en la misma dirección de lo dicho por Skovsmose y Valero (2012) sobre cómo las experiencias de aula de matemáticas, durante la formación inicial de profesores de matemáticas, incorporan una visión interdisciplinar de las prácticas matemáticas propias. Estos autores reportan una experiencia desarrollada por Vithal et al (1997), en la que se concluye que, en un aula de clase inclusiva, los estudiantes que se están preparando para ser profesores, a partir de las prácticas de enseñanza de su profesor, "[...] desarrollaron una serie de proyectos de enseñanza-aprendizaje inspirados por un 'enfoque social, cultural y político' (p. 261), que integraba ideas teóricas acerca del trabajo por proyectos, de la educación matemática crítica y de las etnomatemáticas" (citado en Skovsmose y Valero, 2012, p. 19). Como afirma Andrea, los futuros licenciados podrán comprender que las matemáticas tienen un carácter inclusivo si estas sirven para pensar la relación entre matemáticas y convivencia, matemáticas y ciudadanía, matemáticas y ética $(P, F, 3)$.

Lo anterior es posible si se rompe con la neutralidad política que ha tenido la enseñanza de las matemáticas en contextos sociales y escolares como el nuestro. En palabras de Skovsmose y Valero (2012), los procesos de construcción de conocimiento matemático se deben dar dentro de un ámbito cuya organización escolar sea democrática; así, el sujeto a formar y su comunidad 
educativa pueden compartir valores como el respeto, la igualdad, la responsabilidad social y el interés y actuar coordinadamente en relación con los otros miembros de la comunidad (p. 13).

Entre los rasgos que constituyen al sujeto ético-político, desde esta visión intercultural ofrecida por las voces de los profesores, se cuentan los siguientes:

a. Las otras realidades sociales, las de las otras culturas, que se modelan con las matemáticas propias también deben conceptualizarse en el aula de clase (P, M, 2).

b. Desde las matemáticas inclusivas, es posible pensar la formación para el pluralismo $(P, M, 1)$.

c. La enseñanza de las matemáticas se debe hacer considerando los contextos sociales, para que los estudiantes niños o jóvenes construyan críticamente sus propios contextos (EP, M, 4).

d. Se podrían hacer transformaciones sociales desde el aula si se reconoce que hay otras maneras de construir conocimiento matemático (EP, M, 4).

e. Las matemáticas deben posibilitar la construcción de valores como la equidad, la igualdad, el respeto y la solidaridad ( $\mathrm{P}, \mathrm{F}, 3)$.

En conclusión, podemos afirmar desde las voces de los profesores que, al imbricarse el estudiante como sujeto ético-político en prácticas matemáticas más inclusivas en el aula de clase, podrá tener acceso "[...] a los recursos de poder que están conectados con el conocimiento matemático y las competencias matemáticas" (Skovsmose y Valero, 2007, p. 53), para vivir los valores de la igualdad y la justicia social que tanto nos apremian a todos como miembros de la sociedad colombiana.

\section{Comprensión de sí mismo y autorreflex- ión: reflexiones sobre la práctica profe- sional del profesor}

Otra de las conclusiones resultado de la presente investigación, desde las narrativas de los profesores, está relacionada con la transformación de los roles del profesor como un profesional reflexivo y crítico de su práctica docente. Esta transformación no está vinculada con el tipo de sujeto a formar, sino con el quehacer docente ${ }^{5}$, con lo que hace el profesor y por qué hace lo que hace. Es preciso señalar que, a partir del análisis narrativo, se encuentra que la principal transformación se vincula con la comprensión que el profesor tiene sobre sí mismo, sobre el carácter autorreflexivo del quehacer docente, en cuanto sujeto cognoscente que lleva a cabo prácticas sociales para dar sentido a la acción pedagógica. En resumen, en este apartado se pone de manifiesto que la transformación de las prácticas del formador de profesores y de los futuros licenciados genera comprensión sobre el sentido de la profesión 'ser profesor' de matemáticas, sobre su quehacer docente y su desarrollo profesional.

Para el estudiante Juan, por ejemplo, haber estado en una situación de crisis frente a no encontrar sentido a la profesión 'ser profesor' de matemáticas y comprender cómo se estaba formando lo hizo reflexionar sobre su propia práctica. Las interacciones sociales en clase y los procesos de negociación de significado lo condujeron a cambiar sus concepciones sobre los roles del profesor y del estudiante. Juan resalta la importancia que tuvo en este proceso la manera como los colectivos de profesores se organizaron para desarrollar su práctica profesional, para consensuar una metodología de clase. También, ciertos profesores en particular, durante el desarrollo de sus clases, le mostraron que era posible otro hacer matemático:

Por la forma en que quieren dar a entender ciertos conceptos o cierta materia en la universidad, que es

\footnotetext{
Se entiende por quehacer docente las prácticas profesionales que desarrolla el profesor, como "[...] la competencia de una comunidad de prácticos que comparten, en palabras de Dewey, las tradiciones de una profesión. Comparten convenciones de acción que incluyen medios, lenguajes e instrumentos distintivos" (Schön, 1992, p. 41).
} 
posible que los mismos estudiantes construyan su propio conocimiento matemático, que no hay necesidad de que todo lo estén dando. (EP, M, 4, 39)

Este cambio conceptual y metodológico en las prácticas se puede explicar desde la incidencia de distintos factores relacionados con una organización escolar democrática. En esta última, se definen en colectivo, desde el principio, las tareas que se abordarán en clase, las normas, los valores, las reglas de participación, las formas de trabajo, lo que es válido y no, las valoraciones de los aprendizajes, entre muchos otros aspectos. Para la profesora Andrea, no es un asunto de tener buenas o malas respuestas, sino de interpretar la solución argumentada que provee un compañero en clase, de saber socializar, de regular el proceso creativo $(\mathrm{P}, \mathrm{F}, 3)$.

Este planteamiento corresponde con una organización escolar democrática cuando, según Skovsmose y Valero (2012), el buen funcionamiento de la enseñanza y aprendizaje están relacionados con actores y factores, con el desarrollo de buenas prácticas profesionales: "[...\} por ejemplo, el conocimiento profesional, las creencias, el interés y la reflexión del profesor sobre su propia práctica están asociados a profesores individuales [...]" (Perry, 1998, p. 124, citado en Skovsmose y Valero, 2012, p. 20).

Por otra parte, se encuentra que, tanto para los profesores como para el estudiante de profesor, una organización escolar como la mencionada, durante el desarrollo de las prácticas docentes, promueve la reflexión sobre y en la acción pedagógica. Es a partir de las comunidades de práctica, de los colectivos, como se dota de sentido y significado a dicha acción pedagógica. En su narrativa, el profesor Marcelo señala que promover un aprendizaje durante las prácticas implica generar actitudes críticas, dialogantes entre los mismos estudiantes a través de los saberes matemáticos $(P, M, 2)$. En consecuencia, durante las prácticas de aula, los futuros licenciados, bajo la orientación de su profesor tutor, aprenden a razonar pedagógicamente sobre los contenidos de la enseñanza, así como a valorar por qué y para qué.

De lo anterior, podemos concluir que cuando establecemos relaciones dialógicas en clase compartimos responsabilidades en el trabajo individual y colectivo; exponemos ante los demás nuestras propias reflexiones sobre la práctica. Comprendemos, siguiendo a Taylor (1985), que "[...] los sentidos de las prácticas son intersubjetivos" (Taylor, 1985b, p. 36, citado en McEwan, 2005, p. 253). Es decir, la comprensión que se tiene de sí mismo es el resultado de la comprensión que han conseguido los demás y que ocurre por la escolarización.

El estudiante Juan manifiesta que, con la reflexión sobre su propio hacer matemático en las clases en la universidad, concluyó que las matemáticas son herramientas para comprender el mundo y que esto fue posible porque los mismos profesores formadores promovieron cambios en las concepciones sobre lo que es la matemática, la resolución de problemas y la didáctica (EP, $M$, 4). Él aprendió a reflexionar sobre la acción a partir del análisis de la actuación de profesores que mostraron mayor competencia sobre la profesión, sobre la metodología de clase, sobre lo que ponían en juego.

Incluso, manifiesta en su narrativa el profesor Darío que se puede aprender a 'ser profesor' de otro modo, durante las prácticas, cuando con los colegas se comparten teorías, experiencias, textos, artículos, etc. ( $P, M, 1)$, lo que influye sobre la manera como se investiga en el aula, cómo se sistematiza la propia experiencia, y deviene en asumir una postura reflexiva y crítica sobre la profesión. De acuerdo con lo que plantea McEwan, se aprende a razonar pedagógicamente "[...] ante todo observando, conversando y trabajando con los colegas" (2005, p. 30).

Al narrar lo vivido durante el desarrollo de las prácticas, respecto a lo que sucede en el aula con o a otros, al compartir las experiencias exitosas o fallidas se produce una perspectiva de desarrollo profesional, se reorientan las metas, se adoptan otras estrategias metodológicas, se institucionaliza 
el saber para que todos los estudiantes hagan su proceso de retroalimentación, etc. Estas reflexiones sobre la práctica pueden mejorar la enseñanza y sugerir nuevas posturas, retos y desafíos en la enseñanza de los saberes matemáticos. En su narrativa, el profesor Darío manifiesta que, desde las matemáticas escolares, se pueden cultivar, desarrollar y estimular unos valores distintos, lo cual reafirma su compromiso con la formación de los estudiantes; dichos valores, por ejemplo, están relacionados con los derechos humanos de los ciudadanos. Estas son maneras de ejemplificar que se puede ser profesor con una perspectiva sociopolítica en educación matemática. Prácticas como estas, como dice Shulman (1987), describen la enseñanza como una actividad interpretativa y reflexiva; una actividad en la que los profesores dan vida al currículo y a los textos que enseñan con sus valores y sentido (Shulman, 1987, citado en Gudmundsdottir, 2005, p. 57).

En su narrativa, el estudiante Juan manifiesta que al reflexionar sobre el sentido de las matemáticas durante la enseñanza sus estudiantes también pudieron dar sentido a las matemáticas desde su perspectiva o su propio contexto. Para Julián, por su parte, solo si conocemos la historia de los saberes y los contextos sociales en los cuales se producen podremos adaptar una enseñanza más acorde a las necesidades de los estudiantes, a su realidad social. Concluye que, como estudiante de la licenciatura, su preocupación por vincular los contextos sociales a la enseñanza le permitió comprender que 'ser profesor' es difícil, porque tiene que enfrentarse a situaciones escolares complejas, porque enseñar implica conocimientos distintos de la materia y de las realidades sociales y, además, debe adquirir la competencia para enfrentarse a lo incierto en el aula (EP, $M, 4)$.

Lo anterior se asocia con la percepción de Aronowitz (1993, citado en Skovsmose y Valero, 2012) de asumir la vida "[...] no como una situación estática de 'ser' sino como un 'proceso de llegar a ser'” (p. 11); de llegar a 'ser profesor' dándose cuenta de lo que le falta hacer y llevándolo a cabo en la práctica para concretarlo en resultados. En este proceso de llegar a 'ser profesor' de niños o jóvenes, señala la profesora Andrea que es pertinente darles herramientas a los futuros licenciados para que sean responsables de ese tipo particular de formación y no solo transmitirles informaciones sobre contenidos:

[...] que ellos se ubiquen en que están trabajando con personas, con poblaciones particulares; que no son allá las matemáticas, sino que es un contexto alrededor; que piensen para qué enseñar matemáticas. (P, F, 3, 90-92)

Lo anterior, entonces, se debería vincular con una formación que responda a las demandas de la sociedad, para la asunción de valores para la vida democrática. Para conseguir este propósito, manifiesta en su narrativa el profesor Marcelo que la formación en matemáticas para la ciudadanía debe ofrecerse a lo largo de toda la formación inicial ( $\mathrm{P}, \mathrm{M}, 2)$, para producir un efecto de conjunto y no una escisión entre los saberes matemáticos y los que se vinculan con el ejercicio de la ciudadanía. Una formación en matemáticas para la ciudadanía, siguiendo a Skovsmose y Valero (2012), para que tanto profesores formadores como futuros licenciados y estudiantes de la escuela se "[...] comprometan en una experiencia reflexiva y deliberativa de aprendizaje y enseñanza" (p. 19), durante el desarrollo de las prácticas profesionales.

Como señala la profesora Andrea, esta manera crítica de asumir nuestras reflexiones sobre la práctica implica:

[...] volver a leer las prácticas, leer lo que ya está recortado y yo creo que lo que resumiría es el repensarme siempre y problematizar siempre lo que me pasa y cada cosa que yo me piense para el aula. $(\mathrm{P}$, F, 3, 125-127)

En palabras de Domingo (2011), al referirse al profesor como un profesional que reflexiona sobre su propia práctica, 
[...] cuando hablamos del profesor nos estamos refiriendo a alguien que se sumerge en el complejo mundo del aula para comprenderla de forma crítica y vital, cuestionándose sobre sus propias creencias y planteamientos, proponiendo y experimentando alternativas y participando en la reconstrucción permanente de la realidad escolar. (p. 5)

Al empoderar a los futuros licenciados, Andrea manifiesta que se debe reflexionar más sobre asuntos de pedagogía y ciudadanía que solamente de matemáticas ( $\mathrm{P}, \mathrm{F}, 3)$. Por lo tanto, para ella, los ideales de cambio social desde las aulas deben comenzar por formar a los futuros licenciados para implicarlos en la reflexión sobre o en la acción docente:

Cada cosa que ocurre, que eso es como lo que yo siento que deberían o lo deseable que yo quisiera que mis estudiantes para profesores hicieran, porque siento que si no tienen esa práctica reflexiva ni siquiera van a impactar en lo social o en las matemáticas mismas. (P, F, 3,127-128).

De lo contrario, cuando sean profesores, replicarán el modo en que fueron enseñados en la primaria o secundaria.

Se puede concluir que si los profesores o los futuros licenciados no se apartan de la racionalidad técnica e instrumental de la enseñanza de las matemáticas y sus prácticas se mantienen como en el pasado, ellos:

No darán lugar a tener prácticas reflexivas, ni siquiera impactarán en lo social o en las matemáticas mismas. (P, F, 3, 128)

Esta demanda está relacionada con lo que dice Schön (1992), en su planteamiento sobre la epistemología de la práctica, cuando sostiene que "[...] nuestras percepciones, apreciaciones y creencias tienen sus raíces en los mundos que nosotros mismos configuramos y que terminamos por aceptar como realidad. En su conjunto, los prácticos están permanentemente comprometidos con aquello que Goodman (1978) denomina 'la construcción del mundo'" (p. 44). Mundos posibles en los que las prácticas reflexivas del profesor o de los futuros licenciados se conviertan en acciones de cambio social, empezando por las transformaciones que se piensan desde las mismas aulas.

Algunos de los rasgos que caracterizan acciones de transformación de las prácticas son reconocidos en las voces de los profesores y del estudiante para profesor como conocimientos en o sobre la acción en la profesión 'ser profesor/a' de matemáticas:

a. Para un profesor que enseña matemáticas, su propósito debe ser que los futuros ciudadanos comprendan el mundo con las matemáticas (EP, M, 4).

b. Para pensar el modelo de un profesor crítico, hay que hacer la apuesta política por la transformación de las prácticas (P, M, 1).

c. Valorar la formación ciudadana con las matemáticas ( $\mathrm{P}, \mathrm{M}, 1)$.

d. Orientar la enseñanza de las matemáticas hacia la ética del cuidado del Otro $(\mathrm{P}, \mathrm{M}, 1)$.

e. Debemos hacer una ruptura con el modelo neoliberal y pasar al humanismo $(\mathrm{P}, \mathrm{M}, 1)$.

f. La enseñanza de las matemáticas se debe hacer considerando los contextos sociales, para que los estudiantes niños o jóvenes construyan críticamente sus propios contextos (EP, M, 4).

g. De cada problemática social, a través de la modelación matemática, se pueden construir nuevos conocimientos matemáticos, personalizados y contextualizados (EP, M, 4).

h. Se sigue privilegiando lo disciplinar sobre lo pedagógico y lo didáctico $(\mathrm{P}, \mathrm{F}, 3)$.

i. La historia de la enseñanza de la matemática universitaria sigue teniendo peso en la formación de los profesores (P, F, 3).

j. La formación matemática es para transformar las prácticas de aula (P, F, 3).

k. Para llevar a cabo transformaciones sociales desde el aula, hay que orientar a reflexiones 
sobre pedagogía y didáctica de las matemáticas $(P, F, 3)$.

I. La historia personal del profesor, a partir de las prácticas matemáticas, tiene que posibilitar cambios en la vida de los jóvenes y niños $(\mathrm{P}, \mathrm{F}, 3)$.

$\mathrm{m}$. La resolución de problemas en comunidades de práctica se orienta a la formación de ideales $y$ valores (P, F, 2).

n. Debemos proponer tareas en el marco de proyectos de investigación sobre la formación de los futuros licenciados y analizar los efectos de dichas tareas (P, M, 2).

Ante este abanico de posibilidades de transformación de las prácticas, no hay que olvidar que los programas de formación inicial o continua no solo se deben preocupar por ofrecer a los futuros licenciados herramientas para la conceptualización del saber para enseñar, sino ante todo conectar los saberes con acciones políticas que conduzcan a la comprensión de sí mismos como productores de sentido para su propia práctica y para las acciones de los demás miembros de la comunidad educativa.

Lo anterior, para no caminar sobre el ámbito de la certeza, sino dar lugar a la duda, a inquirir sobre lo que conocemos como parte de nuestras acciones profesionales. Como afirma Schön (1992), "[...] se trata de que nuestra creencia en la legitimidad de nuestro conocimiento se pone a sí mismo en tela de juicio" (p. 23). Solo de esta manera avanzaremos en la búsqueda de vínculos entre la profesión 'ser profesor' y las responsabilidades sociales, lo que no es exclusivo de otras profesiones.

\section{Referencias}

Arboleda, L. (2015). Francisco Vera en Colombia. Transición de las matemáticas del ingeniero a las matemáticas profesionales. En: Desarrollo histórico de las matemáticas y la ingeniería en Colombia en los siglos XIX y XX (pp.151-170). Bogotá: Gente Nueva.
D’Ambrosio, U. (2000). Las dimensiones políticas y educacionales de la etnomatemática. En: A. Martinon (coord.), Las matemáticas del siglo XX. Una mirada en 101 artículos. Recuperado de http://www.sinewton.org/numeros/numeros/43-44/Articulo90.pdf

D’Ambrosio, U. (2007). La matemática como ciencia de la sociedad. En J. Giménez, D. Palomar y M. Civil (2007) (coords.), Educación matemática y exclusión (pp. 83-102). Barcelona: Grao.

Domingo, A. (2011). El profesional reflexivo. Recuperado de http://www.practicareflexiva. pro/wp-content/uploads/2011/05/D.SCHON FUNDAMENTOS.pdf

Elias, N. (2009). Interrelaciones de entramados: problemas de vinculaciones sociales. En Sociologia fundamental (p. 173). Barcelona: Gedisa.

Elias, N. (2012). El proceso de la civilización: Investigaciones sociogenéticas y psicogenéticas. México D.F.: Fondo de Cultura Económica.

García, G. (1996). Reformas en la enseñanza de las matemáticas escolares: perspectivas para su desarrollo. EMA, 1(3), 195-206. Recuperado de http://funes.uniandes.edu.co/1025/1/15 Garc\%C3\%ADa1996Reformas RevEMA.pdf

Gómez, A. (2014). Historia social de la educación matemática en Iberoamérica. 50 años de reformas en el currículo colombiano de matemática en los niveles básico y medio de educación. Revista Unión, 38, 155-176. Recuperado de https://dialnet.unirioja.es/servlet/ articulo?codigo $=4870043$

Goñi, J. (2010). La aspiración a la ciudadania y el desarrollo de la competencia matemática. En J. G. Calleja y M. C. Goñi (eds.), Educación matemática y ciudadanía (pp. 11-58). Barcelona: Grao.

Goodman, L. A., \& Magidson, J. (1978). Analyzing qualitative/categorical data: Log-linear models and latent-structure analysis (pp. 471-473). Cambridge, MA: Abt Books.

Gudmundsdottir, S. (2005). La naturaleza narrativa del saber pedagógico sobre los contenidos. En 
E. McEwan y K. Egan (comps.), La narrativa en la enseñanza, el aprendizaje y la investigación (pp. 52-71). Buenos Aires: Amorrortu.

Guerrero, N. (2016). Tramas narrativas de la enseñanza de la matemática y mejoramiento de la raza en la sociedad colombiana. Revista Científica, 24, 73-82. DOI: http://10.14483/udistrital.jour.RC.2016.24.a7

Hernandez, J. (2000). Una mirada hacia las "matemáticas modernas". Números, 43-44, 209304. Recuperado de http://www.sinewton.org/ numeros/numeros/43-44/Articulo60.pdf

McEwan, E. (2005). La narrativa en el estudio de la docencia. En: E. McEwan y K. Egan (comps.), La narrativa en la enseñanza, el aprendizaje y la investigación (pp.236-259). Buenos Aires: Amorrortu.

Mennell, S. (2001). The Other Side of the Coin: Decivilizing Processes. En: T. Salumets (ed.), Norbert Elias and human interdependencies (pp. 32-49). Quebec: McGill-Queen's University Press.

Quintero, M. (2017). Triple mimesis. Construcción de la trama narrativa: propuesta de metodología hermenéutica (PINH). En Usos de las narrativas. Epistemologías y metodologías: aportes para la investigación. Documento en proceso de publicación

Ricoeur, P. (2006). La vida: un relato en busca de narrador. Ágora, 25(2), 9-22. Recuperado de http://relal.org.co/ media /218/relal.vcb.com.co/images/eventos/
XXIV Retiro de Votos Perpetuos/Documentos/Hno. Luis Bolivar/La Vida en busca de narrador Ricoeur.pdf

Rojas, C. (2001). Violencia y civilización. Bogotá: Publicaciones Universidad Javeriana.

Sánchez, C. y Albis, V. (2012). Historia de la enseñanza de las matemáticas en Colombia. De Mutis al siglo XXI. Quipu, 14(1), pp. 109-157. Recuperado de http://www.revistaquipu.com/ $\underline{\text { Sub1/D3A8TIA/2012/14-1-28415.pdf }}$

Schön, D. (1992). La preparación de profesionales para la demanda de la práctica. En: D. Schön, La formación de profesionales reflexivos ( $p p$. 17-32). Barcelona: Paidós.

Skovsmose, O y Valero, P (2012). Rompimiento de la neutralidad política: el compromiso crítico de la educación matemática con la democracia. Disponible en red en: http:// funes.uniandes.edu.co/2001/1/Skovsmose2012Rompimiento.pdf

Skovsmose, O. y Valero, P. (2007). Educación matemática y justicia social. En: J. Giménez, D. Palomar y M. Civil (coords.), Educación matemática y exclusión (pp.45-62). Barcelona: Grao.

Vanegas, Y. y Giménez, J. (2011). Futuros profesores de matemáticas y ciudadanía. Recuperado de https://www.academia.edu/422640/Futuros profesores de matem $\% \mathrm{C} 3 \% \mathrm{~A} 1$ ticas y ciudadan $\% \mathrm{C} 3 \% \mathrm{ADa}$

White, H. (2003). El texto histórico como artefacto literario y otros escritos. V. T. Lavagnino (trad.). Barcelona: Paidos. 